\title{
Obesidade e transtornos mentais: construindo um cuidado efetivo
}

\author{
Obesity and mental disorders: building an effective care
}

Isabela Azeredo Melca*, Sandra Fortes

\begin{abstract}
Resumo
Os processos de saúde e adoecimento são influenciados por fatores biológicos, psicológicos e sociais. A obesidade é comumente associada aos transtornos mentais, como depressão e ansiedade. Esta associação é constatada em ambas as direções. Transtornos mentais, como os alimentares, a depressão e ansiedade, favorecem o desenvolvimento da obesidade, assim como a obesidade aumenta a incidência dos transtornos mentais. Quando ocorre a ruptura da estabilidade e da homeostase pelo estresse crônico e fatores determinados geneticamente, pelo meio ambiente e pelas relações afetivas e suporte psicossocial, há uma sobrecarga alostática. Através desta sobrecarga alteram-se múltiplos sistemas e surgem doenças clínicas, como hipertensão, diabetes, síndrome metabólica e também doenças psíquicas, tais como a depressão e a ansiedade. O diagnóstico dos transtornos mentais é feito pela combinação de sintomas, queixas somáticas e psíquicas. Queixas físicas por vezes inexplicáveis e sintomas genéricos como humor depressivo, tristeza, impaciência, irritação, inquietação, além da alteração do sono e do apetite, podem estar presentes em diversos transtornos físicos ou mentais. Essa combinação muitas vezes dificulta o correto diagnóstico em pacientes obesos. Os profissionais da atenção básica devem alertar-se para queixas físicas e para os sofrimentos psíquicos presentes em quadros orgânicos. A compreensão dos efeitos fisiopatológicos do estresse e dos transtornos mentais no adoecimento físico reforça a necessidade de que o cuidado efetivo às doenças crônicas não transmissíveis, incluindo a obesidade, seja organizado a partir de modelos que possam abordar também estes aspectos. O tratamento necessita ser integral para que o cuidado seja realmente efetivo. Deve ser centrado na pessoa, na família e na comunidade ao invés de se organizar ao redor da doença. Isso é fundamental no tratamento da obesidade, pois esta se relaciona diretamente a hábitos de vida, estado emocional e nível de estresse, dependendo de uma transformação do indivíduo e de sua forma de viver para que se alcance a resposta desejada. Através dessa abordagem, a atenção básica pode promover a saúde e prevenir agravos.
\end{abstract}

Descritores: Obesidade; Transtorno da compulsão alimentar; Carga alostática; Atenção primária à saúde.

\begin{abstract}
Health and disease processes are developed due to biological, psychological and social determinants. Obesity is usually associated with mental disorders, such as depression and anxiety. This association is evidenced in both directions. Mental disorders, such as depression, anxiety and eating disorders, contribute to the development of obesity, as well as obesity increases the incidence of mental disorders. When stability and homeostasis are disrupted, due to chronic stress and factors such as genetics, environment, affective relationships and psychosocial support, allostatic overload is evidenced. Through this overload, multiple systems are misbalanced and clinical diseases, such as hypertension, diabetes and metabolic syndrome, and mental disorders, such as depression and anxiety, occur. The diagnoses of mental disorders are made based on a combination of somatic and psychiatric symptoms. Unexplained physical complaints and generic symptoms like depressed mood, sadness, impatience, irritability and restlessness are the most frequent ones, together with sleep and appetite disturbances, caused both by physical and mental disorders. These factors often make the correct diagnosis in obese patients more difficult. The primary care professionals must be alert to physical complaints and psychological distress present in organic disorders. The understanding of physiopatological effects of stress and emotional suffering in somatic disorders reinforce that effective care in chronic diseases, such as obesity, have to be organized including these aspects. Treatment needs to be comprehensive so that care can be effective and physical and mental disorders can be properly solved. New therapeutic approaches must be focused on the person, the family and the community instead of focused on the disease. This is essential in the treatment of obesity as it is directly associated with lifestyle, emotional state and stress level, which depends on a change of the individual and his ways of living to be solved. Through this approach, primary care may promote health and prevent diseases.
\end{abstract}

Keywords: Obesity; Binge-eating disorder; Allostasis; Primary health care. 


\section{Introdução}

A moderna neurobiologia vem clareando os mecanismos daquilo que a experiência clínica há muito já sabia: os transtornos mentais compartilham, além de estruturas, mecanismos fisiopatogênicos com as enfermidades crônicas não transmissíveis. Assim sendo, não se pode mais pensar e agir baseado na velha máxima da formação tradicional biomédica de que a psicogênese só é importante quando todos os possíveis mecanismos biológicos foram excluídos na compreensão da etiologia dos processos de saúde e doença. Toda doença física tem componentes psíquicos e sociais. Toda ação terapêutica necessita ser integral para que o cuidado seja realmente efetivo. Este é o paradigma que norteia esta revisão.

\section{A relação entre transtornos mentais e obesidade}

A obesidade vem sendo associada aos transtornos mentais, especialmente ditos transtornos mentais comuns, como a depressão e a ansiedade. Essa associação é constatada em ambas as direções, pois se os transtornos mentais favorecem o desenvolvimento da obesidade, também a obesidade parece aumentar a incidência de depressão, transtorno bipolar, transtorno do pânico ou

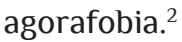

É importante entender que a depressão e a ansiedade são transtornos que compartilham com a obesidade os processos de adoecimento das doenças crônicas não transmissíveis, em especial as cardiovasculares e o diabetes mellitus, o que aumenta o risco de morte prematura.

Como entender essa interação? McEwen ${ }^{3,4}$ e outros autores ${ }^{5,6}$ construíram um modelo explicativo dos mecanismos neurobiológicos presentes nos processos de adoecimento crônico, que demonstra como o estresse, a ansiedade e a depressão se interligam com as principais doenças crônicas não transmissíveis, relacionando a síndrome metabólica e suas principais consequências, diabetes, obesidade e hipertensão, com os transtornos mentais mais prevalentes, a ansiedade e a depressão. Denominada sobrecarga alostática, a partir da definição de Sterling e Eyer de alostase como "a manutenção da estabilidade e da homeostase através da mudança”, que se relaciona com normalidade, esta sobrecarga se faz presente nos processos de adoecimento e representa os custos trazidos ao organismo em geral e ao cérebro em particular, no decurso de tentar manter o equilíbrio na presença de situações de estresse. Esse desgaste, por isso denominado sobrecarga, envolve fatores determinados geneticamente e pelo meio ambiente, incluindo relações afetivas e o suporte psicossocial. Quando ativado por situações de estresse biopsicossocial crônico, este sistema alostático se torna sobrecarregado e diferentes transtornos, inter-relacionados, são iniciados.

Seus mecanismos envolvem a desregulação do eixo neuroendócrino-imunológico, incluindo o sistema nervoso autônomo e o eixo hipotálamo-hipofisário-adrenal, ou seja, no nível central envolvem hipocampo, córtex pré-frontal, amígdala e o tronco cerebral. Assim sendo, podemos entender que mecanismos fisiopatológicos subjacentes a estes processos de adoecimento são biopsicossociais por definição, onde estresse, experiências traumáticas, hábitos de vida, estados emocionais, padrões vinculares de relacionamento e as representações culturais interagem com cargas genéticas, processos inflamatórios, levando a desregulação de sistemas neuroimunoendócrinos.

É importante entender que este processo de sobrecarga se desenvolve como parte do processo de adoecimento. Estímulos diversos geram esta sobrecarga, entre os quais se destacam desde predisposição genética até o contexto social e as dificuldades de vida que aquele indivíduo passou e está passando no momento em que procura cuidado médico. O consequente desequilíbrio físico e psíquico decorrente deste processo, com surgimento da síndrome metabólica, obesidade, hipertensão e diabetes, se associa a quadros depressivos e ansiosos.

Muitos estudos investigaram a relação entre a obesidade e a depressão. Luppino e colaboradores $^{7}$ observaram que $55 \%$ das pessoas obesas possuem maior risco de desenvolverem depressão ao longo da vida, enquanto $58 \%$ das pessoas deprimidas tendem a se tornar obesas.

Mas quais seriam os aspectos deste processo mais especificamente presentes na obesidade? A obesidade é vista pelo corpo como um processo inflamatório, uma vez que o ganho de peso ativa as vias inflamatórias ${ }^{8} \mathrm{e}$ a inflamação ativa citoquinas 
pró-inflamatórias que contribuem para o quadro depressivo. ${ }^{9}$ A obesidade, ao desregular o eixo hipotálamo-pituitário-adrenal (HPA), produz um aumento na secreção de cortisol, o que provoca oscilações do humor. A obesidade central, relacionada principalmente às doenças cardiovasculares, está associada diretamente a distúrbios emocionais, pesadelos e uso de antidepressivos. ${ }^{710}$ Além disso, a medida da circunferência abdominal e o índice de massa corporal (IMC) de pessoas com sobrepeso e obesas vêm sendo associados à maior probabilidade de sintomas do transtorno depressivo maior, moderado e grave." Como já citado, a relação depressão-obesidade é bidirecional, e é presenciada principalmente em mulheres. ${ }^{12,13}$ Assim sendo, o transtorno depressivo pode causar ganho ponderal principalmente pela desregulação neuroendócrina. ${ }^{7}$ Björntorp ${ }^{14}$ afirmou que a depressão provoca obesidade abdominal devido à ativação contínua do eixo HPA e que o cortisol, na presença de insulina, inibe enzimas lipídicas em um processo mediado pelos receptores glicocorticoides.

Porém, como a associação existente é bidirecional, a obesidade também predispõe à depressão e alguns de seus mecanismos são os efeitos negativos da autoimagem, os comentários pejorativos sobre o peso e as consequências somáticas desta doença. Nos dias atuais, a magreza, para os padrões sociais e culturais, é considerada o padrão de beleza. Assim, a obesidade pode contribuir para insatisfação corporal e baixa autoestima, ambas consideradas fatores de risco para depressão.

Também a depressão, na maioria dos casos, causa diminuição da motivação e da sensação de falta de energia, muda os hábitos alimentares e acarreta despreocupação com a saúde da pessoa que a vivencia. O indivíduo deprimido realiza menos esportes, seu engajamento em atividades físicas é menor, o que favorece o desequilíbrio entre ingestão alimentar e gasto calórico. As complicações clínicas podem ser explicadas em parte pelo estilo de vida não saudável, presente geralmente nos transtornos depressivos e ansiosos e em pessoas obesas. ${ }^{12,13}$

A associação entre os demais transtornos mentais e a obesidade revelou também uma alta frequência de quadros de ansiedade entre obesos. Outros transtornos mentais mais graves tais como os transtornos bipolares e os alimentares têm sido menos estudados na sua associação com a obesidade, com exceção do transtorno do comer compulsivo (TCC), pela sua associação com gravidade nos quadros de obesidade. Mas todos estes casos, i.e., transtorno de humor bipolar (THB), os ansiosos e o comer compulsivo, compartilham semelhanças com a depressão e apresentam maior prevalência em mulheres. ${ }^{1}$

Os transtornos ansiosos são mais prevalentes em pacientes com obesidade do que com sobrepeso. ${ }^{15}$ Ser diagnosticado com ansiedade aumenta a probabilidade deste paciente tornar-se obeso em, pelo menos, 12 meses. ${ }^{16}$ Sabe-se que essa associação se encontra principalmente em mulheres, igualmente quando comparada ao transtorno de depressão maior. ${ }^{1}$ E, mais uma vez, verifica-se que o mecanismo causal parece ser a disfunção hipotalâmica. . $^{1718}$

Com relação ao THB, os pacientes acometidos por essa doença apresentam maior risco de sobrepeso e obesidade. Os prováveis fatores de risco para esta associação são: a comorbidade com transtornos ansiosos e de compulsão alimentar, o número de episódios depressivos e sua duração, alguns medicamentos, histórico de internação na fase depressiva e sedentarismo. ${ }^{1}$ Outra informação a ser considerada é a que os pacientes obesos e bipolares apresentam maior número de episódios maníacos e depressivos, além de tentarem mais o suicídio. ${ }^{12,13}$ São considerados como mecanismos causais a desregulação do eixo HPA e fatores neurobiológicos como a diminuição do volume cerebral. ${ }^{19}$ Através da técnica da ressonância magnética, foi possível observar que maiores índices de massa corporal eram associados à diminuição da substância branca e do volume do lobo temporal. ${ }^{20}$

Dentre os transtornos alimentares, a obesidade está associada à bulimia nervosa $(\mathrm{BN})$ e ao transtorno de compulsão alimentar periódica (TCAP). Comumente, o TCAP é comórbido a outros transtornos como o depressivo, o ansioso, de abuso de substâncias e de personalidade. ${ }^{20}$ Nos pacientes com TCAP, os principais contribuintes para esta correlação são o desequilíbrio entre a ingesta e o gasto calórico e a comorbidade com transtorno depressivo maior, ou seja, em alguns estudos atinge $51 \%$ da amostra, o que irá contribuir para a alteração do eixo HPA. ${ }^{10}$

Por fim, é importante lembrar que algumas 
medicações psiquiátricas também podem provocar ganho ponderal e alterações lipídicas e metabólicas. Dentre estas medicações encontram-se os antipsicóticos atípicos, estabilizadores de humor e antidepressivos, como os inibidores seletivos da recaptação de serotonina, os tricíclicos e a mirtazapina. O mecanismo causal ainda é desconhecido, porém se acredita que a fome e a saciedade estejam alteradas no uso dessas medicações. ${ }^{19}$

\section{Como identificar os pacientes com}

\section{transtorno mental}

O diagnóstico da depressão é feito na presença de uma combinação de sintomas, que incluem alterações do sono e apetite, humor depressivo, crenças de menos valia e principalmente a falta de interesse e motivação, ${ }^{21}$ além dos dois sintomas centrais da depressão que são a tristeza (humor depressivo, muitas vezes manifesto por choro fácil) e a perda de prazer nas atividades que valorizava em sua vida. Diversas queixas físicas, entre elas as dores musculoesqueléticas e disfunções sexuais, que podem ser consideradas queixas somáticas do sobrepeso, ${ }^{7,22}$ também estão presentes na depressão e costumam ser valorizadas em sua possível origem orgânica, dificultando a detecção do transtorno mental.

Da mesma forma, a ansiedade se apresenta por queixas físicas por vezes inexplicáveis e por sintomas genéricos como impaciência, irritação e inquietação, além da alteração do sono e do apetite. Devido a esta mistura com queixas somáticas, é fundamental que seja ativamente pesquisada a presença de sofrimento emocional, rastreando-se esses sintomas e indagando sobre como o paciente se sente emocionalmente.

O THB é caracterizado por episódios depressivos e maníacos recorrentes. A fase maníaca possui como características, principalmente, o humor elevado ou eufórico, o aumento de energia e a diminuição da necessidade do sono. ${ }^{23}$

Quanto aos dois transtornos alimentares mais associados à obesidade (TCC e BN), em ambos se encontra o consumo excessivo e repetido de grandes quantidades de comida, em curto período de tempo, acompanhado pela sensação subjetiva de perda de controle, seguidos de culpa. ${ }^{24} \mathrm{Na} \mathrm{BN}$, os episódios de compulsão alimentar provocam comportamentos compensatórios inapropriados, como a indução de vômitos. Estes comportamentos têm o objetivo de evitar o ganho de peso pelo excesso calórico ingerido. No TCAP estes comportamentos são ausentes. ${ }^{24}$

\section{O cuidado do paciente obeso dentro da perspectiva integral}

Essa compreensão dos efeitos fisiopatológicos, do estresse e dos transtornos mentais no adoecimento físico reforça a necessidade de que o cuidado efetivo das doenças crônicas não transmissíveis, dentre as quais se inclui a obesidade, seja organizado a partir de modelos que possam abordar também estes aspectos. É necessário estruturar o tratamento dos principais transtornos mentais associados à obesidade, incluindo o uso de psicotrópicos, mas também incluir abordagem ao estresse e ao sofrimento emocional. O tratamento interdisciplinar, ou seja, a combinação entre psicoterapia, suporte nutricional e exercícios físicos, ${ }^{25}$ apresenta bons resultados.

O trabalho com a nutricionista visa abordar hábitos alimentares errôneos, como sobremesas, lanches energéticos calóricos e a fácil disponibilidade e custo barato das refeições rápidas (fast food). ${ }^{26}$ Este trabalho visa superar o reforço que a mídia transmite para alimentos não saudáveis, carente de nutrientes, porém ricos em gorduras, e o consumo de bebidas alcoólicas, atribuindo a esses sensação de bem-estar ou felicidade, mas não suas consequências, como dano à saúde. ${ }^{27}$

O tratamento integrado com a psicologia é vital, uma vez que as emoções como tédio, raiva e depressão influenciam na quantidade alimentar ingerida. Lidando-se com as emoções negativas, há um predomínio na ingesta de comidas de baixo teor nutritivo, porém extremamente calóricas. ${ }^{28}$ Mas é importante lembrar que na obesidade as intervenções integradas potencializam benefícios nos diversos componentes deste processo de adoecimento. Como exemplo, podemos citar os exercícios físicos que, quando feitos três vezes na semana, têm melhores respostas não apenas na obesidade, mas também na ansiedade e sintomas depressivos. Os principais exercícios propostos são caminhar, subir escadas e dançar, sempre como uma possibilidade de diminuir o sedentarismo e mudar hábitos de vida. ${ }^{29}$ Assim sendo, podemos dizer que o tratamento interdisciplinar 
em pacientes com obesidade melhora sintomas físicos e psicológicos, reduz a prevalência e a gravidade de episódios de compulsão alimentar e a insatisfação com a autoimagem. Logo, o tratamento deve ter como meta os sintomas físicos e psicológicos, uma vez que ambos apresentam-se extremamente associados e parecem fazer parte de um círculo vicioso. ${ }^{25}$

O cuidado de pacientes com síndrome metabólica e obesidade deve ser iniciado já na atenção primária (AP), a principal pela promoção da saúde e prevenção de agravos, ${ }^{30}$ onde a detecção precoce, a abordagem de hábitos de vida e o tratamento de menor complexidade tecnológica podem ser efetivados antes que complicações se instalem. A atenção básica, porta de entrada do Sistema Único de Saúde, deve entender como os eventos cotidianos e as condições médicas se apresentam sobrepostas e como essa sobreposição influencia a saúde mental das pessoas, gerando um círculo vicioso que pode ser interrompido a partir da compreensão deste mecanismo e da abordagem integral deste processo de adoecimento, buscando revertê-lo. Queixas e sintomas impactam as percepções subjetivas da doença e os mecanismos de adoecimento, ${ }^{31} \mathrm{e}$ o desequilíbrio físico e psíquico se alimentam mutuamente.

\section{A abordagem centrada na pessoa e o paciente obeso}

Nas últimas décadas, observa-se o desenvolvimento de novas formas de abordagem que se centram na pessoa, na família e na comunidade ao invés de se organizarem ao redor da doença. ${ }^{32}$ Essa mudança paradigmática é fundamental no tratamento da obesidade, pois esta se relaciona diretamente a hábitos de vida, estado emocional e nível de estresse, dependendo de uma transformação do indivíduo e de sua forma de viver para sua resolução. Assim, é necessário que o profissional de saúde se torne um aliado do paciente na transformação de sua vida, muito mais do que aquele que irá resolver o problema (doença) que o atinge. Para poder caminhar junto e apoiar estas transformações, os profissionais necessitam abandonar posturas tradicionais hierarquizadas em prol de condutas que ajudem os pacientes a refletir sobre suas vidas e o que nelas contribuem para seu adoecimento. Esse entendimento neces- sita de um processo de escuta frequentemente ignorado pelos profissionais que se centram na tarefa de ensinar o paciente o que ele deve mudar em sua vida, nos seus hábitos e forma de viver, ignorando o contexto, a história, as representações e o entendimento que aquele indivíduo tem deste processo e que será fundamental para transformá-lo. Superar o modelo tradicional de "ensinar o paciente" ou "educar o paciente" é superar barreiras que instituem relações hierarquizadas, autoritárias e insatisfatórias. Um dos principais passos é utilizar a escuta e entendimento da narrativa que o paciente faz de seu processo de adoecimento, do agravamento e como acha que esse processo pode ser transformado, incluindo hábitos alimentares, atividade física, momentos de ingesta excessiva (binge eating) e padrões de sono. Conhecer a vida do paciente, suas experiências pessoais, seu momento atual, padrões de relacionamento, expectativas, realizações e frustrações é importantíssimo neste processo. A alimentação se relaciona ao prazer, ao alívio da ansiedade e da depressão, e entender como isso se inscreve na vida, nos hábitos e, consequentemente, no corpo de uma pessoa é parte fundamental dos processos terapêuticos.

As abordagens terapêuticas de cunho psicossocial podem ser feitas de forma individual ou em grupo. As principais intervenções são o desenvolvimento de estratégias de colaboração, denominadas "coping", técnicas de relaxamento, consciência corporal, autoconhecimento e técnicas comportamentais, em especial as da entrevista motivacional. Experiências descritas na literatura ${ }^{25,33,34}$ apoiam os grupos como espaços privilegiados para essa abordagem, que incluem as dificuldades na adesão ao tratamento. Realizam-se encontros semanais, geralmente não mais que oito, com atividades que propiciarão reflexão sobre autoestima, ansiedade, depressão, autoimagem, estresse, satisfação, problemas de família, manutenção das dietas e das atividades físicas regulares. Cria-se um espaço onde se compartilham vivências, debatem-se atitudes, insatisfações e desconfortos e se constrói a responsabilidade de cada um sobre a mudança de determinados comportamentos. A opção por grupos paralelos permitiu notar mudanças nas relações entre pais/ responsáveis e pacientes que contribuíram para melhor resposta ao tratamento. É interessante 
destacar experiências exitosas com grupos de pais de crianças com obesidade, pois o trabalho com as famílias é muitas vezes um aspecto importante do tratamento, mesmo para adultos.

Conhecidas as relações entre obesidade e transtornos psiquiátricos, sobressaltam duas importantes questões para o tratamento medicamentoso da obesidade: como a perda ponderal afetará o estado psicológico do paciente, e como o estado psicológico afetará a perda de peso? Apesar de algumas medicações provocarem aumento de peso, ${ }^{19}$ sabe-se que o próprio quadro psiquiátrico favorece o ganho ponderal, como nos casos de transtorno depressivo e de episódios de compulsão alimentar.12,13 Em alguns casos, o simples tratamento da doença psiquiátrica pode facilitar a perda de peso, seja pela remissão da doença de base ou pela regulação da fome e saciedade, como no uso de agentes serotoninérgicos, usados nos quadros ansiosos, depressivos e transtornos alimentares. ${ }^{35}$

Em contrapartida, as intervenções medicamentosas e cirúrgicas para obesidade, ao provocarem perda ponderal, apresentam melhora significativa dos sintomas depressivos, ansiosos e de compulsão alimentar. ${ }^{19,36}$ Provavelmente, o mecanismo responsável pela melhora no estado psicológico do paciente está relacionado à alteração da autoimagem, maior satisfação corporal e à redução do tônus do eixo HPA., ${ }^{712,13}$

\section{Conclusão}

Os transtornos mentais favorecem o desenvolvimento da obesidade e esta aumenta a incidência dos transtornos mentais. Os principais mecanismos seriam a sobrecarga alostática, com a desregulação do eixo HPA, processos inflamatórios, alteração da autoimagem e insatisfação corporal. Todo profissional de saúde deve estar atento à combinação de sintomas somáticos com sofrimento emocional, pois diversas queixas físicas podem estar presentes em quadros mentais e o sofrimento psíquico presente em quadros orgânicos. Ou seja, toda doença física tem componentes psíquicos e sociais, e vice-versa.

A compreensão da interação fisiopatológica entre o adoecimento físico e psíquico permite que se perceba que ambos encontram-se associados e fazem parte de um círculo vicioso. Nesse contexto, abordagem terapêutica passa a ser centrada na pessoa, família e comunidade, visando promover a saúde, prevenir e cuidar tanto da obesidade quanto dos transtornos mentais, que atuam como fatores de risco para as demais doenças, como a hipertensão arterial e a síndrome metabólica, e que acabam sendo incapacitantes.

\section{Referências}

1. Lin HY, Huang CK, Tai CM, Lin HY, Kao YH, Tsai $\mathrm{CC}$, et al. Psychiatric disorders of patients seeking obesity treatment. BMC Psychiatry. 2013 Jan;13:1. http://dx.doi.org/10.1186/1471-244X-13-1

2. Simon GE, Von Korff M, Saunders K, Miglioretti DL, Crane PK, van Belle G, et al. Association between obesity and psychiatric disorders in the US adult population. Arch Gen Psychiatry. 2006 Jul;63(7):82430. http://dx.doi.org/10.1001/archpsyc.63.7.824

3. McEwen BS. Stress, adaptation, and disease. Allostasis and allostatic load. Ann NY Acad Sci. 1998 May 1;840:3344. http://dx.doi.org/10.1111/j.1749-6632.1998.tb09546.x

4. McEwen BS. Allostasis and allostatic load: implications for neuropsychopharmacology. Neuropsychopharmacology. 2000 Feb;22(2):108-24. http://dx.doi.org/10.1016/S0893-133X(99)00129-3

5. Carvalho SR. A carga alostática: uma revisão. Cad Saúde Colet. 2007 Apr-Jun;15(2):257-74.

6. Stewart JA. The detrimental effects of allostasis: Allostatic load as a measure of cumulative stress. J Physiol Anthropol. 2006 Jan;25(1):133-45. http:// dx.doi.org/10.2114/jpa2.25.133

7. Luppino FS, de Wit LM, Bouvy PF, Stijnen T, Cuijpers $\mathrm{P}$, Penninx BW, et al. Overweight, obesity, and depression: a systematic review and meta-analysis of longitudinal studies. Arch Gen Psychiatry. 2010 Mar;67(3):220-9. http://dx.doi.org/10.1001/ archgenpsychiatry.2010.2

8. Shoelson SE, Herrero L, Naaz A. Obesity, inflammation, and insulin resistance.

Gastroenterology. 2007 May;132(6):2169-80. http:// dx.doi.org/10.1053/j.gastro.2007.03.059

9. Bremmer MA, Beekman AT, Deeg DJ, Penninx BW, Dik MG, Hack CE, et al. Inflammatory markers in late-life depression: results from a population-based study. J Affect Disord. 2008 Mar;106(3):249-55. http://dx.doi.org/10.1016/j.jad.2007.07.002

10. Faith MS, Matz PE, Jorge MA. Obesity-depression associations in the population. J Psychosom Res. 2002 Oct;53(4):935-42. http://dx.doi.org/dx.doi. org/10.1016/S0022-3999(02)00308-2

11. Zhao G, Ford ES, Li C, Tsai J, Dhingra S, Balluz LS. Waist circumference, abdominal obesity, and depression among overweight and obese U.S. adults: 
national health and nutrition examination survey 2005-2006. BMC Psychiatry. 2011 Aug 11;11:130. http://dx.doi.org/1186/1471-244X-11-130

12. de Wit LM, Fokkema M, van Straten A, Lamers F, Cuijpers P, Penninx BW. Depressive and anxiety disorders and the association with obesity, physical and social activities. Depress Anxiety. 2010;27(11):1057-65. http://dx.doi.org/10.1002/ da.20738

13. de Wit L, Luppino F, Straten A, Penninx B, Zitman F, Cuijpers P. Depression and obesity: A meta-analysis of community-based studies. Psychiatry Res. 2010 Jul 30;178(2):230-5. http://dx.doi.org/10.1016/j. psychres.2009.04.015

14. Björntorp P. Do stress reactions cause abdominal obesity and comorbidities? Obes Rev. 2001 May;2(2):73-86. http://dx.doi.org/10.1046/j.1467789x.2001.00027.x

15. Verdolin LD, Borner ARS, Guedes Jr RO, da Silva TFC, Belmonte TSA. Comparação entre a prevalência de transtornos mentais em pacientes obesos e com sobrepeso. Sci Med. 2012 Jan-Mar;22(1):15-22.

16. Bodenlos JS, Lemon SC, Schneider KL, August MA, Pagoto SL. Associations of mood and anxiety disorders with obesity: comparisons by ethnicity. J Psychosom Res. 2011 Nov;71(5):319-24. http://dx.doi. org/10.1016/j.jpsychores.2011.03.004

17. Grudnikoff E, Foley C, Poole C, Theodosiadis E. Nocturnal Anxiety in a Youth with Rapid-onset Obesity, Hypothalamic Dysfunction, Hypoventilation, and Autonomic Dysregulation (ROHHAD). J Can Acad Child Adolesc Psychiatry. 2013 Aug;22(3):235-7.

18. Kessler RC, Angermeyer M, Anthony JC, de Graaf R, Demyttenaere K, Gasquet I, et al. Lifetime prevalence and age-of-onset distributions of mental disorders in the World Health Organisation's World Mental Health Survey initiative. World Psychiatry. 2007 Oct;6(3):168-76.

19. Berkowitz RI, Fabricatore AN. Obesity, Psychiatric Status, and Psychiatric Medications. Psychiatr Clin North Am. 2011 Dec;34(4):747-64. http://dx.doi. org/10.1016/j.psc.2011.08.007

20. Wonderlich SA, Gordon KH, Mitchell JE, Crosby RD, Engel SG. The validity and clinical utility of binge eating disorder. Int J Eat Disord. 2009 Dec;42(8):687705. http://dx.doi.org/10.1002/eat.20719

21. Strakowski SM, Adler CM, DelBello MP. Is depression simply a nonspecific response to brain injury? Curr Psychiatry Rep. 2013 Sep;15(9):386. http://dx.doi. org/10.1007/s11920-013-0386-z

22. Milaneschi Y, Corsi AM, Penninx BW, Bandinelli S, Guralnik JM, Ferrucci L. Interleukin-1 receptor antagonist and incident depressive symptoms over 6 years in older persons: the InCHIANTI Study. Biol
Psychiatry. 2009 Jun;65(11):973-8. http://dx.doi. org/10.1016/j.biopsych.2008.11.011

23. Zeschel E, Correll CU, Haussleiter IS, KrügerÖzgürdal S, Leopold K, Pfennig A, et al. The bipolar disorder prodrome revisited: Is there a symptomatic pattern? J Affect Disord. 2013 Nov;151(2):551-6. http://dx.doi.org/10.1016/j.jad.2013.06.043.

24. Heaner MK, Walsh BT. A history of the identification of the characteristic eating disturbances of Bulimia Nervosa, Binge Eating Disorder and Anorexia Nervosa. Appetite. 2013 Jun;65:185-8. http://dx.doi. org/10.1016/j.appet.2013.01.005

25. Carvalho-Ferreira JP, Cipullo MAT, Caranti DA, Masquio DCL, Andrade-Silva SG, Pisani LP, et al. Interdisciplinary lifestyle therapy improves binge eating symptoms and body image dissatisfaction in Brazilian obese adults. Trends Psychiatry Psychother. 2012;34(4):223-33.

26. Costa RC, Gabriel DC, Costa MJC, Gonçalves MCR, de Oliveira SCP, Asciutti LS. Repercussões sociais no hábito alimentar dos obesos. Estud psicol. 2012;29(4):509-18. http://dx.doi.org/10.1590/S0103166X2012000400006

27. Almeida SS, Nascimento PC, Quaioti TCB. Quantidade e qualidade de produtos alimentícios anunciados na televisão brasileira. Rev Saúde Pub. 2002;36(3):353-5

28. Canetti L, Bachar E, Berry E. Food and emotion. Behav Processes. 2002 Nov;60(2):157-64. http:// dx.doi.org/10.1016/S0376-6357(02)00082-7

29. Tock L, Prado WL, Caranti DA, Cristofalo DM, Lederman H, Fisberg M, et al. Nonalcoholic fatty liver disease decrease in obese adolescents after multidisciplinary therapy. Eur J Gastroenterol Hepatol. 2006 Dec;18(12):1241-5. http://dx.doi. org/10.1097/01.meg.0000243872.86949.95

30. Tanaka OY, Ribeiro EL. Ações de saúde mental na atenção básica: caminho para ampliação da integralidade da atenção. Ciênc Saúde Colet. 2009;14(2):477-86.

31. Gask L, Klinkman M, Fortes S, Dowrick C. Capturing complexity: The case for a new classification system for mental disorders in primary care. Eur Psychiatry. 2008 Oct;23(7):469-76. http://dx.doi.org/10.1016/j. eurpsy.2008.06.006.

32. Bayer AAA, Vasco CC, Lopes SRA, Teixeira CJC. Intervenção Grupal em Pacientes com Obesidade e seus Familiares: Relato de Experiência. Psicol Ciênc Prof. 2010;30(4):868-81.

33. Toral N, Slater B. Transtheoretical model approach in eating behavior. Ciênc Saude Colet. 2007 Nov-Dec; 12(6):1641-50.

34. Stewart M, Weston WW, McWhinney IR, McWilliam CL, Freeman TR, Meredith L, et al. Medicina Centrada 
na Pessoa. $2^{\text {a }}$ ed. Porto Alegre: ArtMed; 2010. 376 p.

35. Hainer V, Kabrnova K, Aldhoon B, Kunesova M, Wagenknecht M. Serotonin and Norepinephrine Reuptake Inhibition and Eating Behavior. Ann NY Acad Sci. 2006 Nov;1083:252-69. http://dx.doi. org/10.1196/annals.1367.017
36. Wadden TA, Sarwer DB, Fabricatore AN, Jones L, Stack R, Williams NS. Psychosocial and behavioral status of patients undergoing bariatric surgery: what to expect before and after surgery. Med Clin North Am. 2007 May;91(3):451-69. http://dx.doi. org/10.1016/j.mcna.2007.01.003

Recebido: $\quad$ 19/08/2013.

Revisado: $\quad$ 26/11/2013.

Aprovado: $\quad$ 04/02/2014. 